\title{
Correction to: Thermal analysis for heat transfer enhancement in electroosmosis-modulated peristaltic transport of Sutterby nanofluids in a microfluidic vessel
}

\author{
K. Ramesh ${ }^{1} \cdot$ J. Prakash ${ }^{2}$
}

Received: 5 February 2019/ Accepted: 16 February 2019/Published online: 12 March 2019

(C) Akadémiai Kiadó, Budapest, Hungary 2019

\section{Correction to: Journal of Thermal Analysis and Calorimetry https://doi.org/10.1007/s10973-018-7939-7}

The original article was published with typographical errors in the non-dimensionalization and parameters involved in the equations.

In Ramesh and Prakash [1], it was noticed that Eq. (1) was incorrect. The correct equation is now provided as follows:

$H= \pm\left(d-b \cos ^{2}\left(\frac{\pi}{\lambda}(X-c t)\right)\right)$,

it was wrongly typed $a$ instead of $d$ in [1], where $d$ is the half-width of the channel. With this change, there is a mismatch in Fig. 1. The correct figure is as follows:

In [1], there was a typographical error in non-dimensional parameter $\bar{S}_{\mathrm{ij}}$, the correct expression is $\bar{S}_{\mathrm{ij}}=d S_{\mathrm{ij}} / c \mu_{\mathrm{f}}$, with this the expression is dimensionless. There were two typographical errors in Eqs. (40) and (41) in [1]. The correct expressions are

$$
\begin{gathered}
u+\alpha\left[\left\{1-\beta\left(\frac{\partial u}{\partial y}\right)^{2}\right\} \frac{\partial u}{\partial y}\right]=-1, \frac{\partial \theta}{\partial y}+B h \theta=0, \\
\frac{\partial \sigma}{\partial y}+B m \sigma=0 \text { at } y=h=1-a \cos ^{2} \pi x,
\end{gathered}
$$

$$
\begin{gathered}
u-\alpha\left[\left\{1-\beta\left(\frac{\partial u}{\partial y}\right)^{2}\right\} \frac{\partial u}{\partial y}\right]=-1, \frac{\partial \theta}{\partial y}-B h \theta=0, \\
\frac{\partial \sigma}{\partial y}-B m \sigma=0 \text { at } y=-h=-\left(1-a \cos ^{2} \pi x\right),
\end{gathered}
$$

where $a(=b / d)$ is the wave amplitude ratio, $\bar{\alpha}\left(=\mu_{\mathrm{f}} \alpha / d\right)$ is the non-dimensional velocity slip parameter (later dropped the bar for convenience). Due to the typographical errors in Eqs. (40)-(41) in [1], the correct expression for Eq. (48) in [1] is given by

$\bar{Q}=Q+1-h-\frac{a}{2}$.

The dimensionless quantities $B h=\eta_{\mathrm{h}} d / \kappa_{\mathrm{h}}, B m=\eta_{\mathrm{m}} d / \kappa_{\mathrm{m}}$, $G r_{\mathrm{t}}=\rho_{\mathrm{f}} g \beta_{\mathrm{t}} d^{2} T_{0} / c \mu_{\mathrm{f}}, G r_{\mathrm{c}}=\rho_{\mathrm{f}} g \beta_{\mathrm{c}} d^{2} C_{0} / c \mu_{\mathrm{f}}$ are introduced in [1].

Since only dimensionless parameters were used in [1], the later sections and findings of [1] remain unchanged by the corrected scalings.

The authors would like to apologize for any inconvenience caused.

The original article can be found online at https:// doi.org/10.1007/s10973-018-7939-7.

K. Ramesh

ramesh.katta1@gmail.com

1 Department of Mathematics, Symbiosis Institute of Technology, Symbiosis International University, Pune 412115, India

2 Department of Mathematics, Srinivasa Ramanujan Center, SASTRA Deemed University, Kumbakonam, Tamilnadu 612001, India 


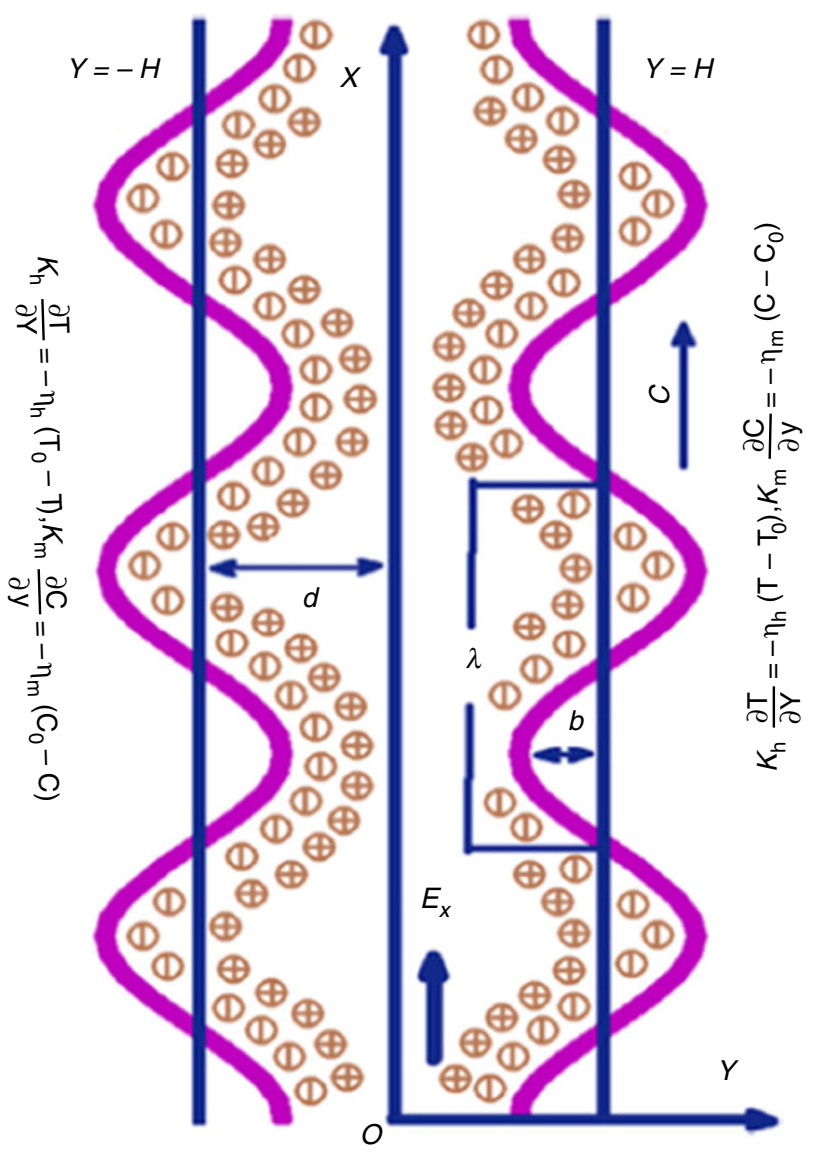

Fig. 1 Physical sketch of the problem

Acknowledgements We wish to thank Professor Asterios Pantokratoras (School of Engineering, Democritus University of Thrace, Greece) for bringing these typographical errors to our attention.

\section{References}

1. Ramesh K, Prakash J (2018) Thermal analysis for heat transfer enhancement in electroosmosis-modulated peristaltic transport of Sutterby nanofluids in a microfluidic vessel. J Therm Anal Calorim. https://doi.org/10.1007/s10973-018-7939-7. 